\title{
Études/Inuit/Studies
}

\section{HURET, Pauline (dir.), 2003 Les Inuit de l'Arctique canadien, Québec, CIDEF-AFI, Collection Francophonie, 266 pages.}

\section{Julie Rodrigue}

Volume 30, numéro 1, 2006

Problématiques des sexes

Gender issues

URI : https://id.erudit.org/iderudit/016161ar

DOI : https://doi.org/10.7202/016161ar

Aller au sommaire du numéro

\section{Éditeur(s)}

Association Inuksiutiit Katimajiit Inc.

Centre interuniversitaire d'études et de recherches autochtones (CIÉRA)

ISSN

0701-1008 (imprimé)

1708-5268 (numérique)

Découvrir la revue

Citer ce compte rendu

Rodrigue, J. (2006). Compte rendu de [HURET, Pauline (dir.), 2003 Les Inuit de l'Arctique canadien, Québec, CIDEF-AFI, Collection Francophonie, 266 pages.] Études/Inuit/Studies, 30(1), 194-196. https://doi.org/10.7202/016161ar d'utilisation que vous pouvez consulter en ligne.

https://apropos.erudit.org/fr/usagers/politique-dutilisation/ 
même si elle est un peu frustrante en raison de ses lacunes. Les illustrations photographiques sont malheureusement de mauvaise qualité et les cartes un peu trop schématiques. La bibliographie témoigne d'une certaine méconnaissance de la littérature européenne francophone, pourtant abondante et importante en ce qui concerne le Paléolithique.

Patrick Plumet 14, allée des Délieuses F78430 Louveciennes France plumet.patrick@free.fr

HURET, Pauline (dir.)

2003 Les Inuit de l'Arctique canadien, Québec, CIDEF-AFI, Collection Francophonie, 266 pages.

Depuis le succès international du film de Zacharias Kunuk, Atanarjuat. La légende de l'homme rapide, les Inuit et leur mode de vie provoquent la fascination. Plusieurs documentaires, articles et livres destinés à un auditoire curieux d'en savoir davantage ont été produits. L'ouvrage collectif intitulé Les Inuit de l'Arctique canadien, dont les textes ont été recueillis par Pauline Huret, s'adresse à la portion francophone de ce public. C'est d'ailleurs, à notre connaissance, la première fois qu'une introduction aussi complète est publiée dans la langue de Molière. C'est donc dans cet esprit qu'il faut aborder cette publication et se demander en quoi elle peut à la fois plaire à un large public et démystifier une réalité qui nous est souvent présentée de façon romantique.

Ce collectif s'ouvre sur une préface signée Michèle Therrien, professeure d'inuktitut et de culture inuit à l'Institut national des langues et civilisation orientales (Inalco) de Paris. Cette préface est suivie de portraits statistiques du Nunavik et du Nunavut. Vient ensuite l'introduction, qui comprend trois textes. Le premier, de l'anthropologue Yvon Csonka, présente l'histoire de l'Arctique central et oriental canadien de la préhistoire au $21^{\mathrm{e}}$ siècle. L'auteur réussit très bien à dresser de façon succincte l'historique du peuplement de cette région. Il termine avec une explication pertinente et indispensable des différences entre le rapport au temps chez les Inuit et chez les historiens occidentaux, ces derniers étant souvent déconcertés par le fait que les Inuit ne datent pas nécessairement les événements. La géographe Béatrice Collignon, quant à elle, explique en quoi la géographie dite savante n'arrive pas à appréhender le territoire inuit d'une façon adéquate et introduit les lecteurs aux perspectives inuit. Vient ensuite l'article de la linguiste Nicole Tersis, à propos de l'inuktitut. L'auteure dresse un historique de l'introduction du syllabaire chez les Inuit puis donne un bref aperçu de la structure syntaxique de l'inuktitut.

La partie suivante porte sur les systèmes de représentations inuit. Elle est également composée de trois textes. Le premier est signé par l'anthropologue Frédéric Laugrand qui présente au lecteur les cosmologies inuit. L'auteur ne se contente pas de dresser un portrait du chamanisme inuit tel qu'il était pratiqué avant l'arrivée des Euro- 
Canadiens. Il explique aussi en quoi la transition vers le christianisme a transformé ces cosmologies, en insistant sur le fait que plusieurs éléments de continuité sont toujours observables. Pauline Huret, diplômée de l'Inalco, présente la notion de personne et comment les individus se perçoivent comme un élément parmi tant d'autres formant un tout qu'elle appelle «cosmos». L'auteure réussi à décrire de façon relativement simple une réalité complexe. Par la suite, Guy Bordin, aussi diplômé de l'Inalco, confronte de façon intéressante les visions occidentales et les représentations inuit de la nuit arctique.

Depuis bien longtemps, le milieu naturel dans lequel évoluent les Inuit suscite la fascination. Deux articles nous présentent comment les Inuit s'approprient ce milieu. Le premier, signé Vladimir Randa, anthropologue et chargé de cours à l'Inalco, explore les relations qu'entretiennent les Inuit avec les animaux qui peuplent leur territoire. Depuis une période de dépendance totale envers les gibiers terrestres et marins jusqu'à l'époque contemporaine où la chasse semble devenir un luxe, il explique en quoi ces relations changent. Le vêtement fait aussi partie de l'adaptation des Inuit à leur milieu naturel. Cécile Herrmann, diplômée de l'Inalco, propose un article sur la confection, l'esthétique et la symbolique des vêtements inuit. Elle y démontre bien en quoi cette activité féminine occupe encore le temps de plusieurs femmes.

Comment aborder l'univers inuit sans traiter de la création artistique et de la créativité? Gwénaële Guigon et Aurélie Le Marec, toutes deux diplômées de l'Inalco, cosignent un article portant sur l'art contemporain inuit. Non contentes de présenter aux lecteurs les différentes productions artistiques (la sculpture, le dessin, l'estampe et les arts textiles), elles en profitent pour discuter des critères esthétiques occidentaux qui sont inadéquats pour aborder l'art inuit et se demandent quels seraient les critères inuit. C'est Francine Desbordes, étudiante à l'Inalco, qui présente l'importance cruciale pour la culture inuit de l'appropriation de la production audiovisuelle. Grâce à cette appropriation, les Inuit sont passés de sujets «documentaires» (comme dans le film Nanook of the North) et ont résisté à l'invasion télévisuelle en provenance du Sud, pour devenir des acteurs incontournables de la production télévisuelle et cinématographique.

Cet ouvrage se termine avec une partie consacrée à l'avenir, qui présente un portrait social, politique et économique du Nunavut et du Nunavik. Sylvie Teveny, diplômée de l'Inalco, explique de façon simple et concise le parcours sinueux des revendications territoriales des Inuit du Nunavik et du Nunavut. Yohann Cesa, doctorant en anthropologie à l'Université Laval (Québec), dresse un portrait de la situation économique des populations du Nunavik et du Nunavut. Il démontre que, bien que plusieurs Inuit soient en partie dépendants des prestations fiscales venant du Québec et/ou d'Ottawa, la réciprocité et le partage ont toujours leur place et leur nécessité au sein des communautés. Ensuite, l'avocat Pierre Rousseau traite de justice chez les Inuit. Il montre comment les Inuit procédaient à la résolution de conflits avant l'implantation du système judiciaire canadien, comment s'est faite la transition vers ce système et comment, plus particulièrement au Nunavut, les contrevenants sont maintenant jugés. Selon Rousseau, malgré des adaptations du système judiciaire en place comme, par exemple, la participation de la communauté par le biais des juges de paix et des aînés, les Inuit font davantage face à une tentative d'assimilation à un 
système judiciaire canadien inefficace qu'au développement d'un véritable système de justice inuit. Céline Petit, doctorante en ethnologie à l'Université de Paris X-Nanterre et à l'Université Laval (Québec), introduit habilement le lecteur à la complexité de la socialisation des enfants avant l'implantation d'un système scolaire qallunaaq. Elle poursuit ensuite avec l'implantation et le fonctionnement de ce système importé. Son texte nous fait aisément comprendre en quoi ces deux façons de transmettre des connaissances sont en contradiction, mais pourtant essentielles. De façon unanime, les Inuit accordent de l'importance à la scolarisation des enfants, mais en soulignant que la transmission des savoirs inuit reste primordiale

Puisque la parution de cet ouvrage a été rendue possible grâce à la participation du Comité international des études françaises et du dialogue des cultures (CIDEF), une dernière partie est consacrée à la francophonie en émergence au Nunavut et au Nunavik. Des francophones, appelés aussi Uiviit (ceux qui disent «oui-oui»), sillonnent l'Arctique canadien depuis longtemps. On y apprend que si peu d'Inuit du Nunavut apprennent le français, il n'en est pas de même au Nunavik où de plus en plus de parents inscrivent leurs enfants au cursus scolaire francophone afin qu'ils obtiennent la maîtrise d'une troisième langue.

Finalement, il faut saluer la généreuse place qu'accorde ce livre à la participation étudiante, autant dans la rédaction des articles qu'au sein du comité de lecture. Bien que l'ouvrage dresse un portrait assez complet des Inuit de l'Arctique canadien, on aurait pourtant aimé y retrouver, sans verser dans le misérabilisme, un texte sur les situations sociales précaires vécues par plusieurs comme, par exemple, la crise du logement, les dépendances et la violence, qui font malheureusement partie du paysage de ces communautés. Dans l'ensemble, nous recommandons ce livre à ceux et celles qui aimeraient s'informer sur le passé et le présent des Inuit du Nord canadien. Il pourrait d'ailleurs constituer un excellent support pédagogique pour un cours d'introduction à l'anthropologie des Inuit.

Julie Rodrigue CIÉRA Université Laval Québec (Québec), G1K 7P4 Canada julie.rodrigue@ciera.ulaval.ca 